\title{
ON THE PROJECTIVE COVER OF AN ORBIT SPACE
}

\author{
K. K. AZAD and GUNJAN AGRAWAL
}

(Received 13 April 1987)

Communicated by J. H. Rubinstein

\begin{abstract}
In this paper, we obtain the projective cover of the orbit space $X / G$ in terms of the orbit space of the projective space of $X$, when $X$ is a Tychonoff $G$-space and $G$ is a finite discrete group. An example shows that finiteness of $G$ is needed.

1980 Mathematics subject classification (Amer. Math. Soc.): 54 H 15.

Keywords and phrases: Stone space, Stone-Cech compactification, projective cover, orbit space.
\end{abstract}

\section{Introduction}

An action of a topological group $G$ on a topological space $X$ is a continuous map $\theta$ from $G \times X$ to $X$ satisfying $\theta(e, x)=x$ and $\theta\left(g_{1}, \theta\left(g_{2}, x\right)\right)=\theta\left(g_{1} g_{2}, x\right)$, where $g_{1}, g_{2} \in G$ and $e$ is the identity of $G$ : a topological space together with a given action is called a $G$-space. A subspace $Y$ of $X$ is called invariant if $\theta(G \times Y) \subseteq Y$, that is, $Y$ becomes a $G$-space with the action induced by $\theta$. Denote $\theta(g, x)$ by $g \cdot x$. For $x \in X$, the set $G_{x}=\{g \cdot x \mid g \in G\}$ is called the orbit of $x$. The collection of orbits is denoted by $X / G$ and the topology on it is coinduced by the map $p$ from $X$ to $X / G$ taking $x$ to its orbit $G_{x}$. The space $X / G$ is called the orbit space of $X$ (with respect to $G$ ). The map $p$ will be called the orbit map. It is open and if $G$ is compact, it is closed as well.

The complete Boolean algebra of regular closed sets of a space $X$ is denoted by $R(X)$ and its Stone space by $S(R(X))$. Denoting the closure and the interior of a set $A$ in $X$ by $\mathrm{Cl} A$ and $\operatorname{Int} A$, respectively, we recall that a set $F$ in $X$ is

(C) 1989 Australian Mathematical Society 0263-6115/89 $\$ A 2.00+0.00$ 
regular closed if $F=\mathrm{Cl} \operatorname{Int} F$, the complement $F^{c}$ of $F$ in $R(X)$ is $\mathrm{Cl}(X-F)$ and the meet $F_{1} \wedge F_{2}$ of regular closed sets $F_{1}$ and $F_{2}$ of $X$ is $\operatorname{Cl}\left(\operatorname{Int} F_{1} \cap \operatorname{Int} F_{2}\right)$. The Stone-Cech compactification of $X$ is denoted by $\beta X$. The map $g$ from $S(R(\beta X))$ to $\beta X$ taking a maximal filter $\mathscr{F}$ of regular closed sets of $\beta X$ to $\cap \mathscr{F}$ (the singleton $\bigcap \mathscr{F}$ is identified with the point in it) is known to be continuous and irreducible. The projective cover of $X$ is the pair $(E(X), h)$, where $E(X)=$ $g^{-1}(X)$ and $h: E(X) \rightarrow X$ is the restriction of $g ; E(X)$ is called the projective space of $X$. Projective covers have been constructed using different methods by Gleason (1958), Rainwater (1959), Strauss (1967), Banaschewski (1968) and Hager (1971).

Recently, Srivastava (1987) has extended an action of a discrete group $G$ on a space $X$ to an action on $\beta X$, which keeps $X$ invariant. In Section 2 of this paper, after introducing an action on $S(R(X))$ through the given action of a discrete group $G$ on $X$, and passing to the extended action on $\beta X$, we find $E(X)$ to be an invariant subspace of $S(R(\beta X))$; thus $E(X)$ becomes a $G$-space. We study the projective cover of an orbit space in Section 3. It is obtained that, in case $G$ is finite, $S(R(X / G))$ is homeomorphic to $S(R(X)) / G$. Since $E(X)$ is $S(R(X))$ if $X$ is compact, $E(X) / G$ is homeomorphic to $E(X / G)$ for a compact $G$-space $X$ with $G$ finite. Taking $X$ to be an arbitrary Tychonoff $G$-space, and passing to its Stone-Cech compactification we establish $E(X) / G$ to be homeomorphic to $E(X / G)$ with the application of the same result obtained for a compact space, to $\beta X$. Finally, an example is provided to show that the orbit space of the Stone space of $R(X)$ need not be homeomorphic to the Stone space of $R(X / G)$.

For terms not explained here we refer to Willard (1970), Bredon (1972) and Walker (1974).

\section{Action on $E(X)$}

Let $Z$ be a zero-set of a $G$-space $X$. Then for $a \in G, a \cdot Z=\{a \cdot z \mid z \in Z\}$ is also a zero-set of $X$. It is easily seen that for a $z$-ultrafilter $\mathscr{F}$ on $X$, the family $a \cdot F$ consisting of $a \cdot Z, Z \in \mathscr{F}$, is a $z$-ultrafilter on $X$. Taking $G$ to be a discrete group, the map $\psi: G \times \beta X \rightarrow \beta X$ given by $\psi(a, \mathscr{F})=a \cdot \mathscr{F}$ defines an action of $G$ on $\beta X$ keeping $X$ invariant [see Srivastava (1987)].

In a similar way, the action on a $G$-space $X$, where $G$ is a discrete group, gives rise to an action $\nu$ of $G$ on $S(R(X))$. In fact, $\nu: G \times S(R(X)) \rightarrow S(R(X))$ is a map which sends $(a, \mathscr{F})$ to $a \cdot \mathscr{F}=\{a \cdot F \mid F \in \mathscr{F}\}$. If $\mathscr{F}$ is a maximal filter of regular closed sets of $\beta X$, then $a \cdot \mathscr{F}$ is also a maximal filter of regular closed sets of $\beta X$ such that $\bigcap a \cdot \mathscr{F}$ is the point $a \cdot p$ in $\beta X$, where $p$ is $\bigcap \mathscr{F}$. Noting 
that $X$ is an invariant subspace of $\beta X$, we have

2.1 LEMMA. $E(X)$ is an invariant subspace of $S(R(\beta X))$.

\section{The orbit space $S(R(X)) / G$}

Throughout this section, unless stated otherwise, $G$ will denote a compact group. Let $X$ be a $G$-space and let $p: X \rightarrow X / G$ be the orbit map. Then, for $\mathscr{F} \in S(R(X))$, the collection $\{p(F) \mid F \in \mathscr{F}\}$ is denoted by $p(\mathscr{F})$. We state the following Lemma without proof.

\subsection{LEMMA. We have}

(i) $p(F) \in R(X / G)$, whenever $F \in R(X)$;

(ii) $p^{-1}(H) \in R(X)$, whenever $H \in R(X / G)$;

(iii) for $H \in R(X / G), p^{-1}\left(H^{c}\right)=\left(p^{-1}(H)\right)^{c}$;

(iv) $p(\mathscr{F}) \in S(R(X / G))$, whenever $\mathscr{F} \in S(R(X))$.

3.2 Proposition. The map $S(p): S(R(X)) \rightarrow S(R(X / G))$ defined by $S(p)$ $(\mathscr{F})=p(\mathscr{F}), \mathscr{F} \in S(R(X))$, is onto and continuous.

ProOF. Let $\mathscr{H} \in S(R(X / G))$ and let $\mathscr{F}$ be a maximal filter in $R(X)$ containing the filter generated by $p^{-1}(\mathscr{H})$. Then $p(\mathscr{F})=\mathscr{H}$, which proves that $S(p)$ is onto. The continuity of $S(p)$ follows by noting that, for $\mathscr{G} \in S(R(X))$, $H \in p(\mathscr{G})$ if and only if $p^{-1}(H) \in \mathscr{G}$.

3.3 LEMMA. Let $X$ be a $G$-space, where $G$ is a finite discrete group and let $\mathscr{F}, \mathscr{H} \in S(R(X))$. Then $p(\mathscr{F})=p(\mathscr{H})$ if and only if $\mathscr{F}=a \cdot \mathscr{H}$ for some $a \in G$.

PROOF. We prove the necessary part only. Suppose to the contrary that $\mathscr{F} \neq a \cdot \mathscr{H}$, for any $a \in G$. Then, for each $a \in G$, there exists an $F_{a} \in \mathscr{F}$ such that $F_{a} \notin a \cdot \mathscr{H}$. Put $F=\bigwedge_{a \in G} F_{a}$. Then $F \in \mathscr{F}$ and $F \notin a \cdot \mathscr{H}$, for any $a \in G$. Since, for each $a \in G, a \cdot \mathscr{H}$ is a maximal filter, there exists $H_{a} \in \mathscr{H}$ such that $F \wedge a \cdot H_{a}=\varnothing$. Let $H=\bigwedge_{a \in G} H_{a}$. Then, for each $a \in G, F \wedge a \cdot H=\varnothing$, that is $F \cap a \cdot \operatorname{Int} H=\varnothing$. For $h \in \operatorname{Int} H, G_{h} \neq G_{x}$, for any $x \in F$ and hence $p(F) \cap p(\operatorname{Int} H)=\varnothing$. This implies that $\operatorname{Int} p(F) \cap C l p(\operatorname{Int} H)=\operatorname{Int} p(F) \cap p(H)=$ $\varnothing$ and therefore $p(F) \wedge p(H)=\varnothing$. Hence $p(\mathscr{F}) \neq p(\mathscr{H})$.

The above lemma gives rise to an injective map $p_{G}: S(R(X)) / G \rightarrow S(R(X / G))$ defined by $p_{G}\left(G_{\mathscr{F}}\right)=p(\mathscr{F}), G_{\mathscr{F}} \in S(R(X)) / G$. Since $S(p)$ is the composition 
of the orbit map $q: S(R(X)) \rightarrow S(R(X)) / G$ with $p_{G}$, it follows that $p_{G}$ is continuous and onto. From the compactness of $S(R(X)) / G$, we obtain that $p_{G}$ is a homeomorphism. Thus we have the following theorem.

3.4 THEOREM. If $G$ is a finite discrete group and $X$ is a $G$-space, then $S(R(X)) / G$ is homeomorphic to $S(R(X / G))$. In particular, if $X$ is compact then $E(X) / G$ is homeomorphic to $E(X / G)$.

Note that the above theorem determines the projective cover of the orbit space $X / G$ in terms of the orbit space of the projective space of $X$, when $X$ is compact and $G$ is finite. Using this result, we generalize it below to an arbitrary Tychonoff $G$-space.

Let $G$ be a finite discrete group and let $X$ be a $G$-space. Since $X / G$ is a dense subspace of $\beta X / G$, the projective space $E(X / G)$ of $X / G$ is $h_{\beta}^{-1}(X / G)$, where $\left(S(R(\beta X / G)), h_{\beta}\right)$ is the projective cover of $\beta X / G$. In view of Lemma 2.1, $E(X)$ is a $G$-space. Now we have

3.5 THEOREM. Let $X$ be a $G$-space, where $G$ is a finite discrete group. Then $E(X / G)$ is homeomorphic to $E(X) / G$.

Proof. Let $q: \beta X \rightarrow \beta X / G$ be the orbit map. Then the map

$$
q_{G}: S(R(\beta X)) / G \rightarrow S(R(\beta X / G))
$$

defined by $q_{G}\left(G_{\mathscr{F}}\right)=q(\mathscr{F})$, where $\mathscr{F} \in S(R(\beta X))$, describes a homeomorphism [see, Theorem 3.4]. Since, for $\mathscr{H} \in S(R(\beta X)), \bigcap \mathscr{H} \in X$ if and only if $\bigcap q(\mathscr{H}) \in$ $X / G$, it follows that $q_{G}(E(X) / G)=E(X / G)$ and we have the result.

3.6 REMARK. Let $\left(E(X / G), g_{1}\right)$ be the projective cover of $X / G$. Then, in view of Theorem $3.5,\left(E(X) / G, h_{1}\right)$ can be regarded as the projective cover of $X / G$, where $h_{1}$ is the composition of the restriction of the homeomorphism $q_{G}$ to $E(X) / G$ and $g_{1}$. It may also be noted that $h_{1}$ maps an orbit $G_{\mathscr{F}}, \mathscr{F} \in E(X)$, to the orbit in $X / G$ determined by $\bigcap \mathscr{F}$.

3.7 EXAMPLE. Let $D$ be the open interval $(0,1)$ of the real line. Consider the $G$-space $D$, where $G$ is the discrete group consisting of all non-decreasing homeomorphisms from $D$ to $D$ (group operation being the composition of homeomorphisms) and the action $\theta$ of $G$ on $D$ is given by $T \cdot x=T(x), x \in D$, $T \in G$. Let $\mathscr{F}$ be the filter in $R(D)$ generated by the collection consisting of regular closed sets containing $1 / 4$ in their interiors, and closed intervals $[s, 1 / 4]$, $0<s<1 / 4$; and let $\mathscr{H}$ be the filter in $R(D)$ generated by the collection consisting of regular closed sets containing $3 / 4$ in their interiors, and closed intervals $[3 / 4, t], 3 / 4<t<1$. It is easy to check that both $\mathscr{F}$ and $\mathscr{H}$ are in $S(R(D))$ 
and that $\mathscr{F} \neq T \cdot \mathscr{H}$, for any $T \in G$. This shows that $G_{\mathscr{F}}$ and $G_{\mathscr{X}}$ are distinct and both belong to $S(R(D)) / G$, whereas $S(R(D / G))$ is the singleton.

\section{References}

B. Banaschewski (1968), 'Projective covers in categories of topological spaces and topological algebras', General topology and its relations to modern analysis and algebra, III, pp. 63-91 (Proc. Conf. Kanpur).

G. E. Bredon (1972), Introduction to compact transformation groups, (Academic Press, New York).

A. M. Gleason (1958), 'Projective topological spaces', Ilinois J. Math. 2, 482-489.

A. W. Hager (1971), 'The projective resolution of a compact space', Proc. Amer. Math. Soc. 28, 262-266.

J. Rainwater (1959), 'A note on projective resolutions', Proc. Amer. Math. Soc. 10, 734-735.

Kavita Srivastava (1987), 'On the Stone-Cech compactification of an orbit space', Bull. Austral. Math. Soc. 36, 435-439.

D. P. Strauss (1967), 'Extremally disconnected spaces', Proc. Amer. Math. Soc. 18, 305-309.

R. C. Walker (1974), The Stone-Cech compactification, (Springer-Verlag, Berlin).

S. Willard (1970), General topology, (Addison-Wesley, Reading, Mass.).

'Vijaya Niwas'

198, Mumfordganj

Allahabad, 211002

India
Department of Mathematics and Statistics University of Allahabad Allahabad

India 